\title{
Ewing's sarcoma of the mandible misdiagnosed as periodontal inflammation: Report of three cases
}

\author{
Eda Balkaya ${ }^{1}$, Ceyhun Bozkurt ${ }^{1}$, Ali Emre $\mathrm{Aksu}^{2}$, Selahattin Özmen ${ }^{3}$, Sonay İncesoy- \\ Özdemir ${ }^{1}$, Gürses Şahin ${ }^{1}$ \\ ${ }^{1}$ Department of Pediatric Hematology-Oncology, Dr. Sami Ulus Maternity and Children's Health and Diseases Training \\ and Research Hospital; ${ }^{2}$ Department of Plastic Reconstructive and Aesthetic Surgery, Hacettepe University Faculty of \\ Medicine; ${ }^{3}$ Department of Plastic Reconstructive and Aesthetic Surgery, Gazi University Faculty of Medicine, Ankara, \\ Turkey.E-mail: edabalkaya@yahoo.com
}

Received: 2nd March 2017, Revised: 17th April 2017, Accepted: 23th April 2017

SUMMARY: Balkaya E, Bozkurt C, Aksu AE, Özmen S, İncesoy-Özdemir S, Şahin G. Ewing's sarcoma of the mandible misdiagnosed as periodontal inflammation: Report of three cases. Turk J Pediatr 2017; 59: 704-707.

Ewing's sarcoma (ES) is the second most common childhood primary malignant tumor of the bone. The most popular locations of ES are long bones and pelvis. The involvement of the mandible is very rare in childhood. In last 10 years, we met with three cases of ES of the mandible in our department. Initially the patients had symptoms similar to periodontal inflammation. The involvement of the mandible might be considered with periodontal inflammation in its initial stages, what frequently leads to delayed treatment. Although this tumor has an aggressive clinical behavior and rapid growth, early diagnosis can reduce patient's morbidity and mortality and thus it is important to distinguish from periodontal inflammation.

Key words: children, Ewing's sarcoma, jaw, mandible, periodontal inflammation.

Ewing's sarcoma (ES) is a skeletal system malignant tumor which is originated from neurons. ${ }^{1}$ ES accounts for $10 \%$ of all childhood skeletal malignancies. The most common locations of ES are long bones and pelvis (nearly $50 \%) .^{1,2}$ The occurrence of ES in the head and neck region is uncommon and diagnosed in less $3 \%$ of all ES. ${ }^{1,2}$ ES is one of the most aggressive tumors of childhood. Periodontal inflammations are very common; but in the literature, as far as we know, there are small number of ES of the mandible misdiagnosed as periodontal inflammation. ${ }^{2-4}$

We present three cases and the medical records of patients with ES of the mandible who were diagnosed and treated during the last ten years at our clinic. Initially they were considered as periodontal inflammation in the epicenter and their treatment was delayed.

\section{Case Reports}

Case 1

Nine-year-old girl who was diagnosed and treated as periodontal inflammation initially, was referred to our clinic with the progressively growing mass in the jaw at the end of two months. On clinical examination, a fixed expansive mass of approximately $9 \times 8 \mathrm{~cm}$ in diameter was observed on the left side of the mandible and the lower jaw teeth were seen to be shifted. There were no palpable lymph nodes. Her physical examination was otherwise unremarkable. Plain radiography of mandible showed a lytic, expansive mass covering the left side of the mandible, which caused destruction of the mandible (Fig. 1). The temporomandibular computer tomography (CT) scan showed an expanding and destructive mass in size of $8 \times 5 \times 7 \mathrm{~cm}$ on the left part of mandible (Fig. 2, 3). The mass was infiltrated to masseter muscle and adjacent fatty tissue. Incisional biopsy was performed and the pathological diagnosis revealed as ES. Metastasis was not detected with radiological studies. Euro-EWING 99 chemotherapy protocol was administered. Even though five courses of VIDE (vincristine, ifosfamide, doxorubicin, etoposide) was administered, patient's mass grew aggressively. The surgery 
of hemimandibulectomy with disarticulation at the temporomandibular joint was performed and the bony defect was reconstructed with titanium reconstruction plate. After surgery chemotherapy protocol completed: a total seven courses of VIDE and eight courses of VAI (vincristine, actinomycin-D, ifosfamide). Presently, the illness is in remission and the patient is disease-free for 84 months.

\section{Case 2}

Three-year-old girl who was diagnosed and treated as periodontal inflammation initially, was admitted to our department with symptoms of progressively enlarging mass on her chin for two months. On clinical examination a fixed, painless mass of approximately $3 \times 2.5$ $\mathrm{cm}$ in diameter was observed on the mandible corpus and there were several palpable submandibular lymph nodes (max. $1 x 1 \mathrm{~cm}$ of size). Plain radiography showed a lytic mass on the mandible corpus. Temporomandibular computerized tomography scan showed a $3 \times 2 \times 2.5 \mathrm{~cm}$ expanding and destructive mass on the mandible corpus. Then incisional biopsy was performed and the pathological diagnosis revealed ES. Metastasis was not observed with radiological studies. Euro-EWING 99 chemotherapy protocol was administered. After seven courses of VIDE chemotherapy protocol, the malignant tissue was resected and reconstructed with free iliac flap. After operation she was given eight courses of VAI chemotherapy protocol. She is in remission for 96 months.

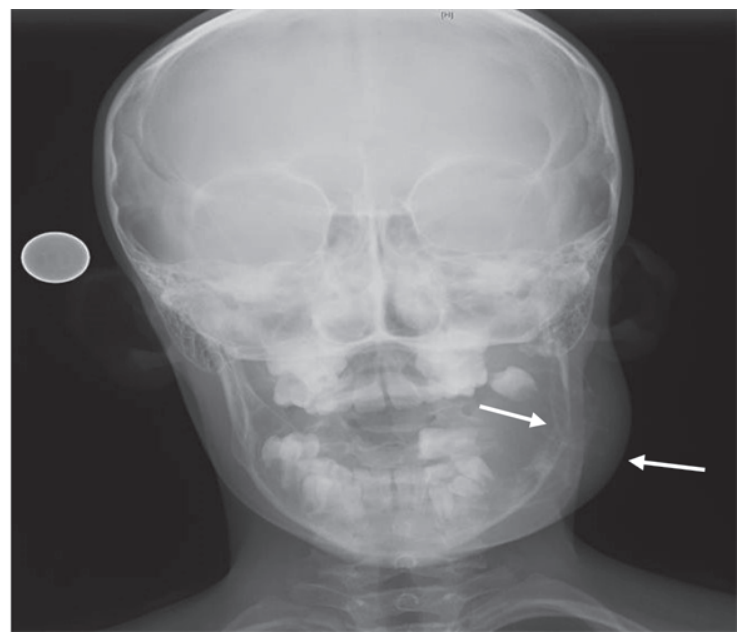

Fig. 1. Direct radiography showing an osteolytic and expansive mass which made erosion and density loss on the left mandible corpus.

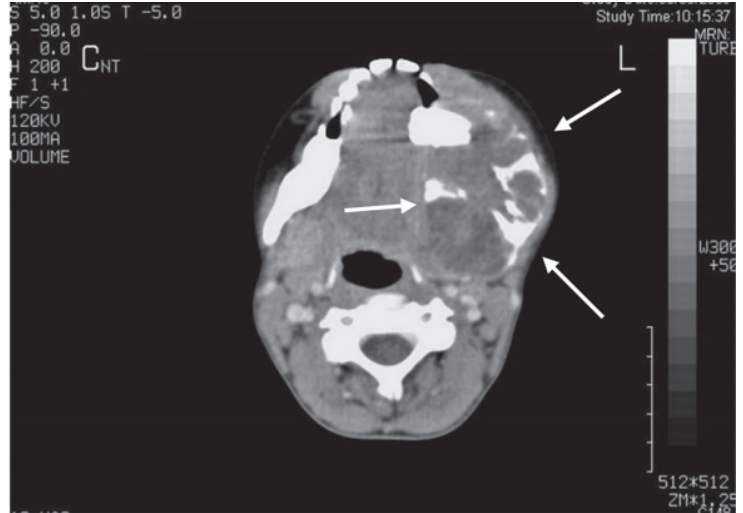

Fig. 2. Mediastinal windows of CT images showing heterogeneous and hypodense mass lesion involving peripheral contrast in the left mandibular corpus.

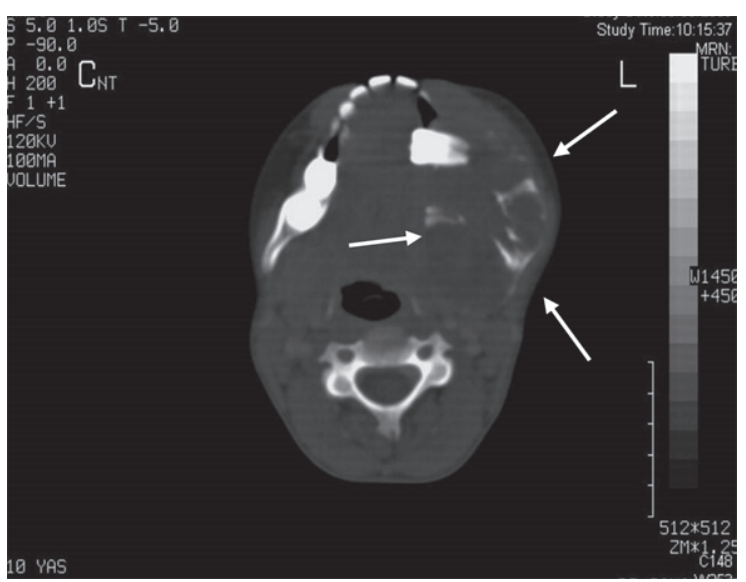

Fig. 3. Bone windows of CT images showing expanding and destructive mass on the left mandible.

\section{Case 3}

A thirteen-year-old girl was admitted to our department due to progressively enlarging mass on the left side of the mandible for two months and we learned that she was diagnosed and treated as periodontal inflammation for two weeks previously. During her clinical examination, she had a painless, fixed mass of approximately $3 \times 2 \mathrm{~cm}$ in diameter on the left side of the mandible and there was a soft tissue swelling extending from the first front incisor teeth to first molar teeth in the gingivobuccal sulcus. There were no palpable lymph nodes. Her physical examination was otherwise unremarkable. Plain radiography of mass showed a $2 \mathrm{~cm}$ radiolucent area on the left ramus of the mandible. Temporomandibular computer tomography showed a $5 \times 6 \mathrm{~cm}$ expansive and destructive mass on the left 
mandible. No metastasis was seen with imaging. Incisional biopsy was performed and the pathological diagnosis revealed as ES. She received Euro-EWING 99 chemotherapy protocol (vincristine, ifosfamide, doxorubicin, etoposide, actinomycin D, cyclophosphamide); but her mass grew progressively and became inoperable. Radiotherapy (RT), at a total dose of $5580 \mathrm{cGy}$, was given to the area of the mass. After radiotherapy, the patient's treatment was maintained with Euro-EWING 99 chemotherapy protocol and distant bone metastases were observed at the sixteen months of treatment. After all relapse treatment regimens, the patient died in 24th month of her treatment due to the progression of her disease. Informed consent form was obtained from the family.

\section{Discussion}

Ewing's sarcoma is the second most common childhood primary malignant tumors of the bone and constitutes $10 \%$ of primary malignant bone tumors., ${ }^{1,4}$ ES is frequently observed between the ages from 5 to 20.3,4 For ES, the most favorable sites are distal extremities and central locations (e.g. skull, clavicle, vertebrae and ribs). ${ }^{1,3,4}$ The occurrence of ES in the head and neck region is uncommon and less than $3 \%$ of all ES.1,2,3,5 The involvement of the mandible is primarily very rare, but when it occurs in the jaw, mandible is more frequently affected than the maxilla. ${ }^{1-5}$

Reported in many cases, the first signs are pain and swelling of the mandible and it can mimic odontogenic inflammation. In the literature, ES involving mandible cases are mostly seen in the reports of dental journals. This shows that ES involved in the mandible cases are often seen by the dentists and considered as periodontal inflammation. So ES involving mandible cases are often misdiagnosed as periodontal inflammation. Number of case reports are limited. ${ }^{2-4}$ When the patients came to our clinic, there were rapidly progressive growth masses in the mandible and the patients were accompanied by pain. Also they were misdiagnosed as periodontal inflammation and their treatment delayed. In addition, loosening teeth, lymphadenopathy, otitis media, paresthesia, fever, weight loss, anemia increased erythrocyte sedimentation rate, increased CRP and leukocytosis may be observed in ES involving mandible cases. ${ }^{2,5,6}$ These findings are not specific, but when there is a prolonged periodontal inflammation, you should point on necessity to do complementary exams for early diagnosis. ${ }^{4}$

If there is a progressive growth mandibular lesion or extensive lytic lesion of the bone tissue, histopathological evaluation should be mandatory, suggesting the possibility of malignant pathology such as ES, neuroblastoma, rhabdomyosarcoma, osteosarcoma and benign pathology such as histiocytosis-X, osteomyelitis. $.5,7,8$ Our cases were positive for CD99, vimentin and desmin with characteristic membranous pattern and negative for other markers, leading to diagnosis of ES.

For ES involving jaw bones, the most common radiological features are the presence of sun-ray spicules of periosteal bone and displacement or destruction of unerupted tooth follicles. ${ }^{1,3}$ In the presented cases, the radiographic findings were osteolytic lesions of the mandible. By some authors, CT scan and magnetic resonance imaging have been considered as the best imaging methods for evaluating the tumor.1,6,7 In our cases, the CT scan was made before treatment, they showed the intraosseous origin of the tumor and expanding destructive mass of mandible. Additionally, only one of them had soft tissue invasion and none of them had metastasis at the time of diagnosis.

Clinically, this tumor has an aggressive behavior characterized by rapid growth and high probability of micro metastasis at diagnosis. Patients usually die within a few months after diagnosis, because of hematogenous spread and lung metastases. ${ }^{1,5,6,8}$ But primary lesions of the head and neck region (especially mandible) have a significantly better survival rate than other sites of ES ${ }^{8}$. At the present time, the 5-year survival rate has increased to approximately $75 \%$ with multidisciplinary treatment protocols for ES involving jaws. $6,8,9$

If ES occurs from the head and neck region, a significant number of patients have no metastases at diagnosis just like our patients. ${ }^{9}$ And in ES of the mandible, the most common metastases regions are other bone tissues. ${ }^{1,4}$ In our study, distant bone metastases had been confirmed only in one of the patient at the sixteen months of the treatment and she 
died in the 24th month of her treatment due to progression of the disease.

In conclusion, if there is a prolonged periodontal inflammation and a progressive growth of the mass in the mandible, radiological assessment should be done firstly. If it reveals an extensive lytic lesion of the bone tissue, histopathological evaluation requires for the early diagnosis of the malignant pathology such as ES. Although this tumor has an aggressive behavior clinically, early diagnosis can reduce patient's morbidity and mortality.

\section{REFERENCES}

1. Lopes SL, Almeida SM, Costa AL, Zanardi VA, Cendes F. Imaging findings of Ewing's sarcoma in the mandible. J Oral Sci 2007; 49: 167-171.

2. Gosau M, Baumhoer D, Ihrler S, Kleinheinz J, Driemel O. Ewing sarcoma of the mandible mimicking an odontogenic abscess-a case report. Head Face Med 2008; $24: 1-5$.
3. Keshani F, Jahanshahi G, Attar BM, et al. Ewing's sarcoma in mandibular similar to dental abscess. Adv Biomed Res 2014; 3: 62.

4. Brazão-Silva MT, Fernandes AV, Faria PR, Cardoso SV, Loyola AM. Ewing's sarcoma of the mandible in a young child. Braz Dent J 2010; 21: 74-79.

5. Sharada P, Girish HC, Umadevi HS, Priya NS. Ewing's sarcoma of the mandible. J Oral Maxillofac Pathol 2008; 10: 31-35.

6. Singh JP, Garg L, Shrimali R, Gupta V. Radiological appearance of Ewing's sarcoma of the mandible. Indian J Radiol Imaging 2003; 13: 23-25.

7. Khanna G, Sato Y, Smith RJ, Bauman NM, Nerad J. Causes of facial swelling in pediatric patients: Correlation of clinical and radiologic findings. Radiographics 2006; 26: 157-171.

8. Mukherjee A, Ray JG, Bhattacharya S, Deb T. Ewing's sarcoma of mandible: A case report and review of Indian literature. Contemp Clin Dent 2012; 3: 494-498.

9. Bernstein M, Kovar H, Paulussen M, et al. Ewing's sarcoma family of tumors: Current management. Oncologist 2006; 11: 503-519. 